09

\title{
Широкополосная фильтрация рентгеновского спектра при прохождении через поликапиллярную структуру
}

\author{
(C) А.Г. Турьянский, С.С. Гижа, В.М. Сенков \\ Физический институт им. П.Н. Лебедева РАН, Москва, Россия \\ E-mail: algeo-tour@yandex.ru
}

Поступило в Редакцию 10 августа 2018 г.

Показана эффективность широкополосной фильтрации полихроматического рентгеновского спектра в поликапиллярной структуре в области энергий фотонов $E>10 \mathrm{keV}$. Фильтром являлся поликапиллярный стержень из боросиликатного стекла длиной $40.5 \mathrm{~mm}$, содержащий $10^{6}$ параллельных капилляров гексагонального сечения. Фильтрация осуществлялась путем отклонения оси капиллярной структуры от направления первичного пучка в диапазоне углов $0-4 \mathrm{mrad}$. В области энергий $12-23 \mathrm{keV}$ достигнуто относительное ослабление спектральной плотности излучения до $17 \mathrm{~dB}$.

DOI: 10.21883/PJTF.2019.01.47151.17498r

Выделение оптимальной спектральной полосы рентгеновского спектра является одной из основных задач различных аналитических методов. Для получения наибольшей спектральной плотности в заданном участке тормозного рентгеновского спектра при электронном облучении мишени обычно прикладывается ускоряющее напряжение, при котором максимальная энергия электронов $E_{m}$ в 2-3 раза выше максимума спектрального распределения рентгеновских фотонов. При возбуждении заданной характеристической линии спектра с энергией $E_{c}$ величина $E_{m} \geqslant(3-4) E_{c}[1,2]$. Поэтому в большинстве практических случаев в рабочем пучке доминирует интегральная составляющая жесткой части спектра, которая создает фоновое рассеяние при прохождении через объект и непосредственно в детекторе излучения. Фильтрация жесткой части жестко коллимированных рентгеновских пучков эффективно осуществляется с помощью рентгеновских зеркал с использованием резкой спектральной зависимости коэффициента отражения от угла скольжения пучка относительно поверхности зеркала [3,4]. Однако ввиду малости углов скольжения они не обеспечивают возможности фильтрации пучков с размером поперечного сечения в плоскости отражения более $1-2 \mathrm{~mm}$. Зеркальные фильтры не могут быть также использованы при получении проекционных изображений объекта от микрофокусного источника в пределах широкого телесного угла.

Спектральная фильтрация в изогнутых капиллярах, образующих поликапиллярные линзы, рассматривалась в работах [5-7]. Для заданной энергии спектра существует пороговое значение радиуса кривизны оси капилляра, при котором происходит полное внутреннее отражение на стенках капилляра [5]. Поэтому спектр излучения у выходной поверхности линзы сильно зависит от радиального расстояния от оси линзы. Однако для потока излучения, распространяющегося под малыми углами относительно оси линзы, спектры первичного и прошедшего излучения практически совпадают, что снижает эффективность фильтрации.

В настоящей работе показано, что наиболее эффективно широкополосная фильтрация спектральной плотности рентгеновского излучения обеспечивается при прохождении излучения через структуру прямолинейных капилляров при оптимальной угловой ориентации оси капилляров относительно направления падающего излучения.

В качестве спектрального фильтра использовался поликапиллярный стержень (далее поликапилляр), изготовленный из боросиликатного стекла, который состоял из набора микрокапилляров, имеющих гексагональную форму поперечного сечения. Стекло содержало примесь $\mathrm{ZrO}_{2}$ с относительным весовым содержанием $\sim 1 \%$. Микрокапилляры были собраны в гексагональные микросборки, содержащие до 400 капилляров. Микросборки находились в стеклянной трубке с толщиной стенок $0.4 \mathrm{~mm}$. Длина стержня составляла $40.5 \mathrm{~mm}$, а внешний диаметр - $5.3 \mathrm{~mm}$.

Определение геометрических параметров поликапиллярной структуры проводилось на сканирующем конфокальном микроскопе MicroTime 200 с использованием лазерного излучения на длине $\lambda=375 \mathrm{~nm}$ и оптическом микроскопе Dino-Lite AM7013MZT4. На рис. 1, $a$ показано оптическое изображение торца поликапилляра, иллюстрирующее структуру гексагональных микросборок. На рис. $1, b$ приведено конфокальное изображение отдельных капилляров в плоскости торца стержня, полученное при сканировании сфокусированным пучком лазера зоны $10 \times 10 \mu \mathrm{m}$. По данным математической обработки средняя величина периода расположения микросборок $L_{s}$ и отдельных капилляров в микросборке $L$ составляют 100 и $4.5 \mu \mathrm{m}$ соответственно. Среднее расстояние между параллельными стенками капилляров $4 \mu \mathrm{m}$. Таким образом, отношение площади сечения канала к общей площади капилляра равно $q=0.79$. 

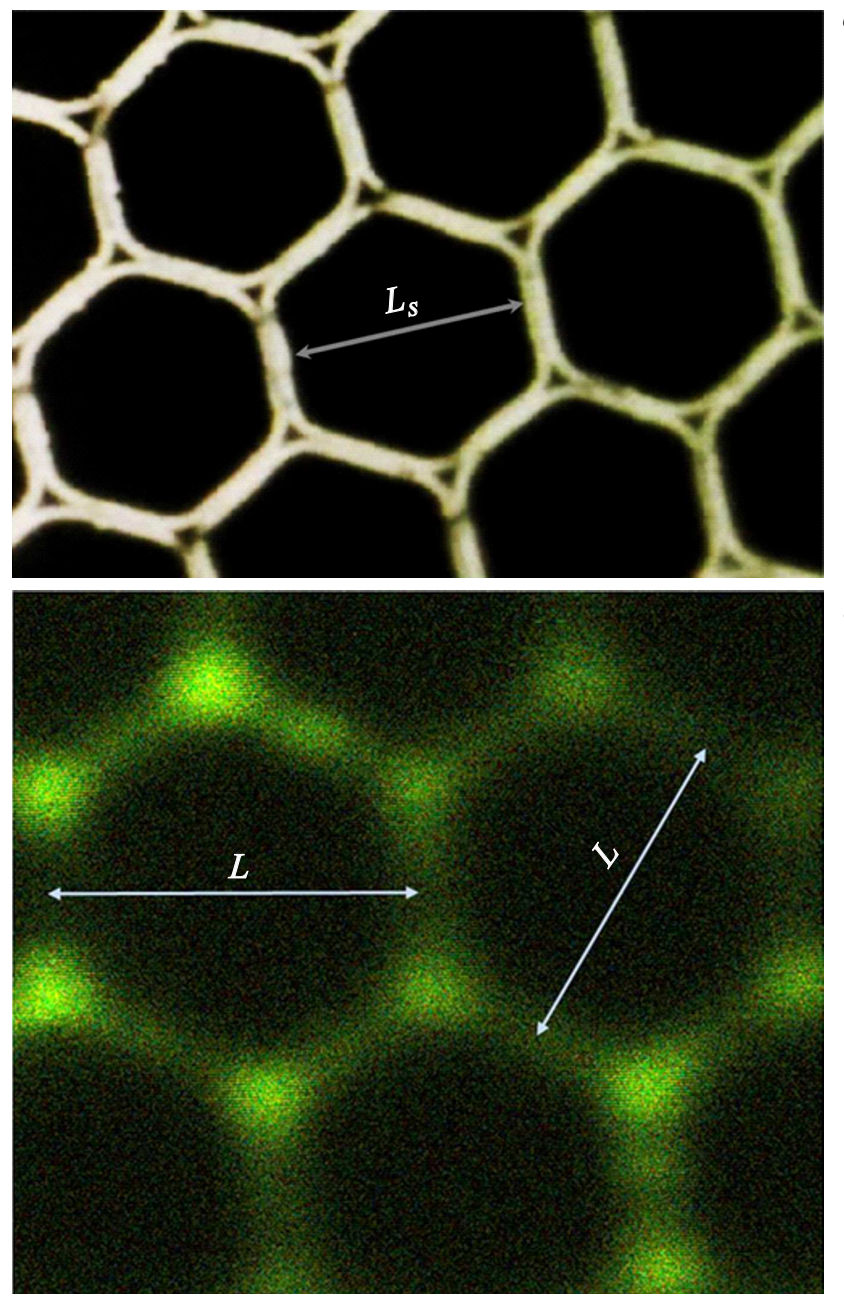

Pис. 1. Изображения торца поликапиллярного стержня. $a-$ структура сборок микрокапилляров, стрелкой указан период микросборок $L_{s}(100 \mu \mathrm{m}) ; b-$ структура отдельных микроканалов (конфокальная микроскопия, поле сканирования $10 \times 10 \mu \mathrm{m})$, стрелками указан период отдельных капилляров в микросборке $L$.

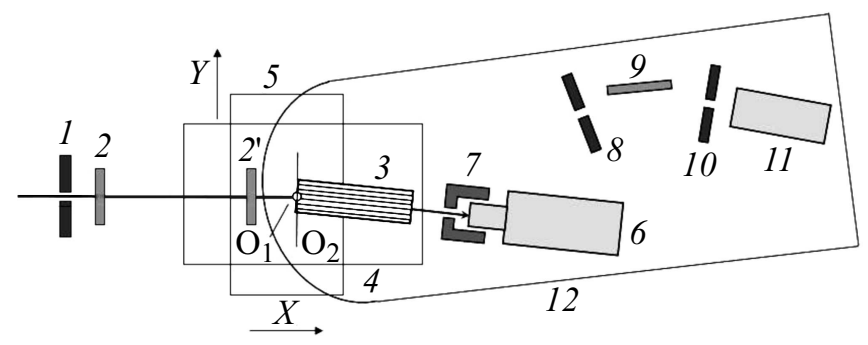

Рис. 2. Общий вид измерительной схемы в горизонтальной плоскости. Пояснение в тексте.

На рис. 2 показана схема измерения спектров пропускания рентгеновского излучения через поликапилляр. Схема собрана на платформе рентгеновского рефлектометра ComplefleX-5 (CDP systems). Максимальное рабочее напряжение на рентгеновской трубке с медным анодом и мощность составляли $40 \mathrm{kV}$ и $300 \mathrm{~W}$ соответственно. Выходная щель коллиматора 1 обеспечивала угловую расходимость в горизонтальной плоскости $0.2 \mathrm{mrad}$. Для ограничения угловой расходимости в вертикальной плоскости устанавливались горизонтальные щели 2 и $2^{\prime}$ шириной от 50 до $250 \mu \mathrm{m}$. Расстояние между щелями составляло $75 \mathrm{~mm}$. Входная поверхность поликапиллярного стержня устанавливалась на оси вращения гониометра с помощью систем позиционирования 4, 5. Измерения рентгеновских спектров проводились полупроводниковым спектрометром 6 (X 123 SDD Amptek), установленным на расстоянии $20 \mathrm{~mm}$ от выходного торца поликапилляра. Площадь входного окна спектрометра составляла $25 \mathrm{~mm}^{2}$, толщина детектирующего кристалла кремния - $0.5 \mathrm{~mm}$, энергетическое разрешение на линии $\mathrm{Cu} K_{\alpha}(8.05 \mathrm{keV})-$ $160 \mathrm{eV}$. Для защиты от рассеянного излучения устанавливался свинцовый коллиматор 7. Угловое позиционирование поликапиллярного стержня 3 осуществлялось путем поворота вокруг вертикальной оси $O_{1}$ и горизонтальной оси $O_{2}$. Для точной установки поликапилляра по оси рентгеновского пучка спектрометр и коллиматор удалялись и путем независимого поворота платформы 12 вводились щелевые диафрагмы 8,10 , сцинтилляционный детектор 11 и кристаллмонохроматор 9, настроенный на спектральную линию $\mathrm{Cu} K_{\alpha 1}$. При установке оси поликапилляра параллельно оси первичного пучка абсолютный коэффициент пропускания на указанной спектральной линии составлял $T=0.51$. С учетом параметра $q$, определяющего отношение площади сечения канала к общей площади капилляра, средний коэффициент пропускания через капилляр равнялся 0.65. При этом угловая расходимость прошедшего через поликапилляр пучка составляла $3 \mathrm{mrad}$.

На рис. 3, a показаны результаты измерения спектров пропускания через поликапилляр для набора углов отклонения $\theta$ оси капилляров от оси прямого пучка. На спектр прошедшего излучения, содержащий тормозное излучение и характеристические линии анода $\mathrm{Cu} K_{\alpha}$ и $\mathrm{Cu} K_{\beta}$, накладываются флуоресцентные линии $\mathrm{Zr}$, входящего в состав используемого стекла. Наиболее резко отсечка спектра наблюдается при значении $\theta=3.9 \mathrm{mrad}$, которое соответствует критическому углу полного внешнего отражения от стенок капилляра для фотонов с энергией $E=7.7 \mathrm{keV}$. При энергии фотонов $E>20 \mathrm{keV}$ начинается рост коэффициента пропускания жесткой части спектра через поликапилляр [8,9]. Это, очевидно, обусловлено переходом от волноводного механизма прохождения излучения через поликапилляр к обычному типу ослабления интенсивности $I$ по экспоненциальному закону

$$
I=\exp [-\mu(1-q) \rho l],
$$

где $\mu$ - массовый коэффициент ослабления стекла, $q-$ отношение площади сечения канала к общей площади 

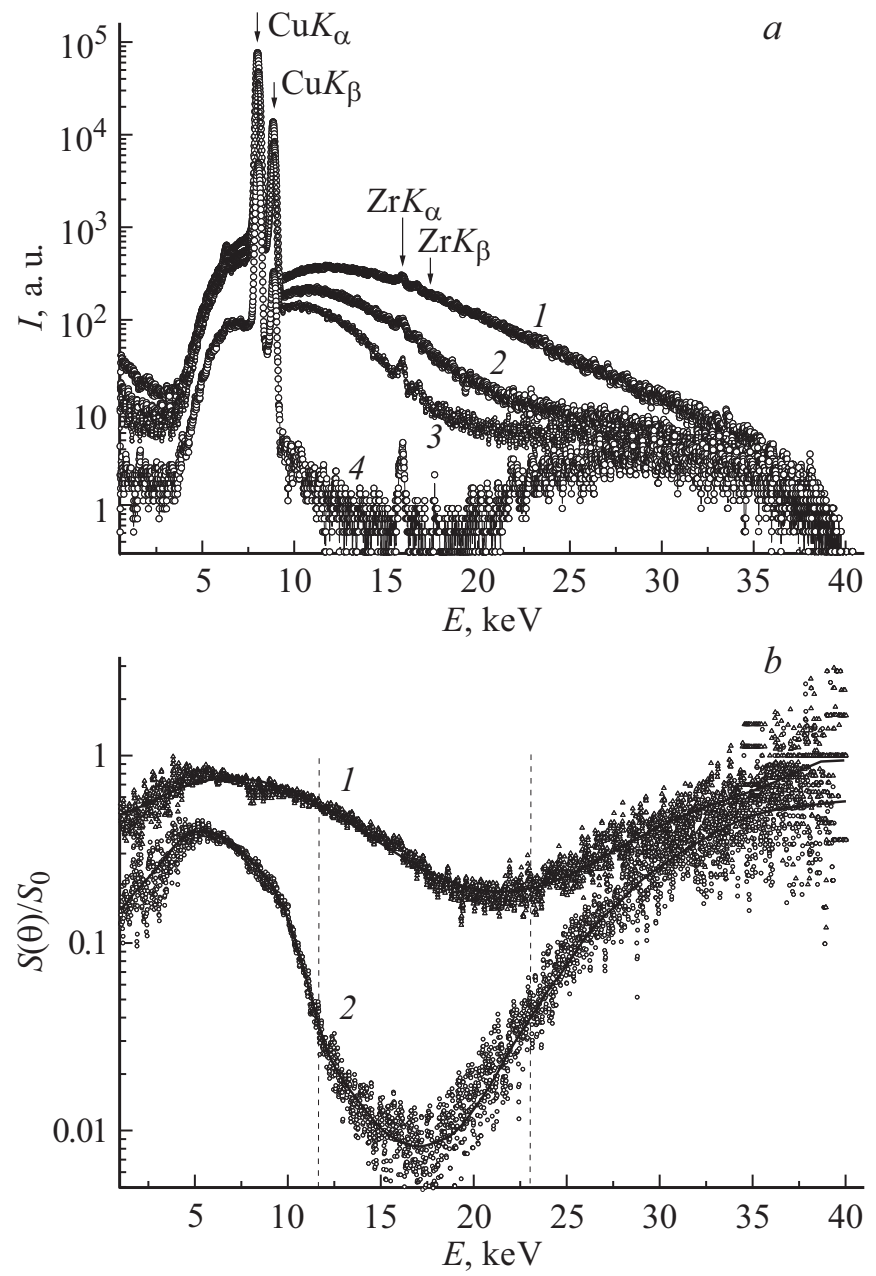

Рис. 3. $a-$ экспериментальные зависимости спектров пропускания от угла $\theta$ между осями капилляров и первичного пучка. $\theta$, mrad: $1-0,2-1.6,3-2.1,4-3.9 . b-$ отношение спектральной плотности излучения $S(\theta) / S_{0}$ при $\theta=1.6(1)$ и $3 \mathrm{mrad}(2)$.

капилляра, $\rho$ - плотность стекла, $l$ - длина пути излучения, равная длине поликапиллярного стержня. При этом необходимо рассматривать усредненное значение плотности поликапилляра, которое равно $\rho(1-q)$. В нашем случае $q=0.79$, что приводит к приблизительно пятикратному снижению средней плотности и радиационному пробою поликапилляра при энергиях $>20 \mathrm{keV}$.

В качестве характеристики, иллюстрирующей эффективность спектральной фильтрации с помощью поликапилляра, может быть принято экспериментальное отношение $S(\theta) / S_{0}$, где $S(\theta)$ и $S_{0}$ - измеренная спектральная плотность рентгеновских фотонов $\Delta N / \Delta E$ при углах отклонения $\theta>0$ и $\theta=0$ соответственно. Очевидно, что при этом не требуется пересчета спектральной плотности с учетом эффективности поглощения в рентгеновском спектрометре, поскольку условия регистрации эквивалентны. При этом осцилляции на эксперимен- тальной зависимости $S(\theta) / S_{0}$ в области спектральных линий меди и циркония были исключены с помощью интерполяции спектральной зависимости тормозной части спектра. Как видно из рис. $3, b$, минимумы спектральной плотности $S(\theta) / S_{0}$ при $\theta=1.6 \mathrm{mrad}$ (кривая 1) и $3 \mathrm{mrad}$ (кривая 2) наблюдаются при энергиях фотонов 22 и $17.3 \mathrm{keV}$ соответственно. При этом максимальное ослабление спектральной плотности в указанных минимумах составляет соответственно 3.8 и 50 раз (5.8 и $17 \mathrm{~dB}$ ). При $\theta=3 \mathrm{mrad}$ ослабление излучения на порядок величины наблюдается в достаточно широком спектральном диапазоне от 11.7 до $23.1 \mathrm{keV}$, показанном на рис. 3, $b$ штриховыми линиями. При энергиях $E>20 \mathrm{keV}$ эффективность фильтрации монотонно снижается. При этом в отличие от мягкой части спектра, проходящей через отверстия капилляров, жесткая часть проходит через полное сечение поликапилляра. Очевидно, что ширина полосы фильтрации может быть увеличена путем сдвига границы радиационного пробоя при включении в состав стекла оптимальной концентрации тяжелых элементов.

В рассмотренной выше схеме поликапилляр является фильтром режекторного типа [10], т.е. резко понижает уровень спектральной плотности излучения прошедшего пучка в широкой полосе $\sim 10 \mathrm{keV}$. Это позволяет, во-первых, резко повысить чувствительность стандартного метода рентгеновского флуоресцентного анализа для химических элементов, спектральные линии которых находятся в полосе режекции. Во-вторых, это повышает контрастность регистрации проекционных изображений биологических или полимерных объектов, для которых оптимальная полоса спектра, несущая информацию о внутренней структуре, находится в диапазоне $E \leqslant 10 \mathrm{keV}$. В этом случае поликапиллярная структура должна размещаться перед 2Dдетектором и обеспечивать фильтрацию жесткой части спектра, создающей преимущественно фоновый сигнал.

\section{Список литературы}

[1] Dyson N.A. X-rays in atomic and nuclear physics. 2nd ed. Cambridge University Press, 2005. 416 p. https://doi.org/10.1017/CBO9780511470806

[2] Рентгенотехника / Под ред. В.В. Клюева. М.: Машиностроение, 1980. Кн. 1. С. 431.

[3] Bridou F., Delmotte F., Delmotte F., Troussel Ph., Villette B. // Nucl. Instrum. Meth. Phys. Res. A. 2012. V. 680. N 11. P. 69 74. https://doi.org/10.1016/j.nima.2012.04.002

[4] Турьянский А.Г., Гижа С.С., Сенков В.М., Савельев С.К. // Письма в ЖТФ. 2014. Т. 40. В. 8. С. 56-63.

[5] Schields P.J., Gibson D.M., Gibson W.M., Gao N. // Powder Diffraction. 2002. V. 17. N 2. P. 70-80. https://doi.org/10.1154/1.1482080

[6] Mazon D., Abadie Q., Dorchies F., Lecherbourg L., Mollard A., Malard P., Dabagov S. // Nucl. Instrum. Meth. Phys. Res. B. 2015. V. 355. N 15. P. 301-306. https://doi.org/10.1016/j.nimb.2015.04.042 
[7] Gao N., Janssens K. Polycapillary $X$-ray optics. // $X$-ray spectrometry: recent technological advances. John Wiley \& Sons, Ltd, 2004. P. 89-110.

[8] Hubbell J.H. // Int. J. Appl. Rad. Isotop. 1982. V. 33. N 11. P. $1269-1290$.

[9] Henke B.L., Gullikson E.M., Davis J.C. // Atom. Data Nucl. Data Tabl. 1993. V. 54. N 2. P. 181-342. http://henke.lbl.gov

[10] Турьянский А.Г., Гижа С.С., Сенков В.М., Пиршин И.В., Станишевский Я.М. // Письма в ЖЭТФ. 2016. Т. 104. В. 6.

C. 430 -434. DOI: $10.7868 / \mathrm{S} 0370274 \mathrm{X} 16180107$ 